\title{
Throughput Optimization via Cooperative Spectrum Sensing with Novel Frame Structure
}

\author{
Hang Hu, ${ }^{1}$ Hang Zhang, ${ }^{1}$ Hong Yu, ${ }^{1}$ and Javad Jafarian ${ }^{2}$ \\ ${ }^{1}$ College of Communications Engineering, PLA University of Science and Technology, Nanjing 210007, China \\ ${ }^{2}$ School of Electrical \& Electronic Engineering, The University of Manchester, Manchester M13 9PL, UK
}

Correspondence should be addressed to Hang Zhang; hangzh_2002@163.com

Received 28 August 2013; Revised 10 November 2013; Accepted 10 November 2013

Academic Editor: Matjaz Perc

Copyright (C) 2013 Hang Hu et al. This is an open access article distributed under the Creative Commons Attribution License, which permits unrestricted use, distribution, and reproduction in any medium, provided the original work is properly cited.

In cognitive radio (CR) networks, cooperation can greatly improve the performance of spectrum sensing. In this paper, we propose a novel cooperative spectrum sensing (CSS) frame structure in which CR users conduct spectrum sensing and data transmission concurrently over two different parts of the primary user (PU) spectrum band. Energy detection sensing scheme is used to prove that there exists an optimal sensing bandwidth which yields the highest throughput for the CR network. Thus, we focus on the optimal sensing settings of the proposed sensing scheme in order to maximize the throughput of the CR network under the conditions of sufficient protection to PUs and required bandwidth for potential CR user data transmission. Some algorithms are also derived to jointly optimize the sensing bandwidth and the final decision threshold. Our simulation results show that optimizing the sensing bandwidth and the final decision threshold together will further increase the throughput of the CR network as compared to that which only optimizes the sensing bandwidth or the final decision threshold.

\section{Introduction}

Cognitive radio is a promising technology to improve the efficiency of spectrum usage [1]. The CR users should obey the two following etiquettes [2]: (1) they are allowed to use some unoccupied spectrum bands; (2) they must vacate the spectrum bands quickly whenever the PUs return. In order to avoid interference with PUs, a CR user needs to efficiently and effectively detect the presence of the PUs. CR users can use one of several common detection methods [3], such as matched filter, feature detection, and energy detection. Energy detection is the most popular method addressed in the literature [4-8]. Measuring only the received signal power, energy detection has much lower complexity than the other two schemes. Therefore, we consider energy detection for spectrum sensing in this paper.

The detection quality of spectrum sensing easily suffers from the fading and shadowing environment, which can cause hidden terminal problem. To combat these impacts, cooperative spectrum sensing has been introduced to obtain the space diversity in multiuser CR networks [9-12]. Game theory is suitable for analyzing conflict and cooperation among rational decision makers [13-16]. In [17], the authors focused on dynamical effects of coevolutionary rules on the evolution of cooperation. Game theory has been widely applied to study distributed optimization problems, such as power control, dynamic spectrum access and management $[18,19]$, and so on. In [20], the authors proposed an evolutionary game framework to answer the question of "how to collaborate" in multiuser decentralized cooperative spectrum sensing. The cooperative spectrum sensing involves sensing, reporting, and decision making steps [21]. In the sensing step, every CR user performs spectrum sensing independently using energy detection method and makes a local decision. In the reporting step, all the local sensing observations are reported to a fusion center (FC). The decision step is made to indicate the absence or the presence of the PU.

In the frame structure of periodic spectrum sensing (PSS), the CR user senses the status of the radio spectrum in the sensing slot and transmits data using the remaining frame duration [22]. Since the CR user must interrupt data transmission during the sensing slot, the CR user transmission delay will be long. Thus, in the case of delay sensitive applications, the QoS (quality of service) will not 


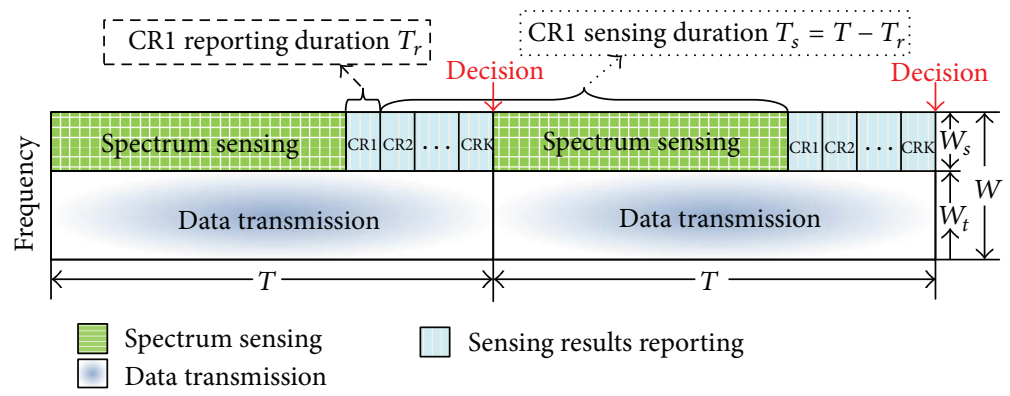

FIgURE 1: Novel frame structure for cooperative spectrum sensing.

be guaranteed. In our previous work [23], we propose a novel cooperative spectrum sensing frame structure in which CR users conduct spectrum sensing and data transmission concurrently over two different parts of the primary user spectrum band. In this way, the CR users do not need to interrupt data transmission in the sensing stage, and the QoS can be guaranteed. The optimal multiminislot sensing scheme and the optimal fusion scheme that minimize the CR user transmission delay were analyzed in [23]. However, the performance of throughput with the novel CSS frame structure was not investigated. In this paper, we focus on analyzing the throughput of the CR network.

In the novel frame structure designed for CSS, one part of the PU transmission bandwidth is assigned exclusively to spectrum sensing and sensing results reporting, and the other part is assigned exclusively to potential CR user data transmission. According to this frame structure, under the condition of sufficient protection to PUs, an increase in the sensing bandwidth results in a lower false alarm probability, which leads to higher throughput of the CR network. However, the increase of the sensing bandwidth results in a decrease of the bandwidth assigned to potential CR user data transmission and hence the throughput of the CR network. Therefore, there could exist the optimal sensing bandwidth that maximizes the throughput of the CR network.

In this paper, we study the tradeoff problem for cooperative spectrum sensing with novel frame structure. We are interested in the problem of designing the sensing bandwidth to maximize the throughput of the CR network under the conditions of sufficient protection to PUs and required bandwidth for potential CR user data transmission. When energy detection is utilized for spectrum sensing, we prove that there indeed exists one optimal sensing bandwidth which yields the highest throughput for the CR network.

In the cooperative spectrum sensing with novel frame structure, we employ the counting rule as the fusion rule at the FC since it requires the least communication overhead and is easy to implement. Since CR is originally designed to improve the spectrum efficiency, maximizing the CR users' throughput is one of the most practical interests. Our object is to find the optimal sensing settings to maximize the throughput of the CR network under the conditions of sufficient protection to PUs and required bandwidth for potential CR user data transmission. To achieve that, we propose an efficient search algorithm that jointly optimizes the sensing bandwidth and the final decision threshold. Computer simulations show that optimizing the sensing bandwidth and the final decision threshold together will further increase the throughput of the CR network as compared to that which only optimizes the sensing bandwidth or the final decision threshold.

The rest of this paper is organized as follows. The cooperative spectrum sensing with novel frame structure is analyzed in Section 2. The optimization problem of maximizing the throughput with sensing bandwidth and final decision threshold as the optimization variables is formulated in Section 3. Optimal sensing settings of throughput are analyzed in detail in Section 4. Simulation results are presented in Section 5, followed by concluding remarks in Section 6.

\section{Cooperative Spectrum Sensing with Novel Frame Structure}

We consider a CR network, with a number of $K$ CR users and a fusion center. The size of the secondary network is small compared with the distance between the primary network and the secondary network to ensure the QoS of primary link. Then the path loss of each CR user is almost identical and the primary signals received at the CR users are considered to be independent and identically distributed (i.i.d.) [24]. In cooperative spectrum sensing, local CR users individually sense the channels and then send information to the fusion center, and the fusion center will make the final decision.

Figure 1 illustrates the novel frame structure designed for cooperative spectrum sensing. In the frequency domain, considering the case that the CR users know the PU transmission bandwidth $W, W$ is divided into two parts, namely, $W_{s}$ and $W_{t}$ $\left(W_{t}=W-W_{s}\right) . W_{s}$ is assigned exclusively to spectrum sensing and sensing results reporting, which means that the status of PU can be decided by sensing a portion of PU bandwidth, and we do not need to sense the whole PU bandwidth. The other part $W_{t}$ is assigned exclusively to potential CR user data transmission. The CR users conduct spectrum sensing and data transmission concurrently over two different parts of the primary user spectrum band.

In the time domain, it is assumed that the frame duration is $T$ and the individual reporting duration is $T_{r}$. Since each CR user continues the spectrum sensing after sending its sensing result to the fusion center, the sensing duration for each CR user is $T_{s}=T-T_{r}$. 
The essence of spectrum sensing is a binary hypothesistesting problem [25]:

$$
\begin{aligned}
& \mathscr{H}_{0}: \text { the PU is absent; } \\
& \mathscr{H}_{1}: \text { the PU is present. }
\end{aligned}
$$

The received signal $r_{i}(k)$ at the $i$ th CR user can be formulated as

$$
r_{i}(k)= \begin{cases}\varepsilon_{i}(k), & \mathscr{H}_{0}, \\ h_{p} s(k)+\varepsilon_{i}(k), & \mathscr{H}_{1}\end{cases}
$$

where $r_{i}(k)$ is the received signal at the $i$ th CR user, $\varepsilon_{i}(k)$ is the noise and $\varepsilon_{i}(k) \sim \mathscr{C} \mathscr{N}\left(0, \sigma^{2}\right), s(k)$ is the signal of PU and $s(k) \sim \mathscr{C} \mathscr{N}\left(0, \sigma_{s}^{2}\right)$, and $h_{p}$ is the channel gain between PU and the $i$ th CR user. The power spectrum density (PSD) of the noise and the PSD of the primary signal are evenly distributed, with values of $S_{n}$ and $S_{p}$, respectively. The SNR (signal-to-noise ratio) of PU's signal at the ith CR user is $\gamma_{i}=\left|h_{p}\right|^{2} \sigma_{s}^{2} / \sigma^{2}$.

Since the primary signals received at the CR users are considered to be i.i.d., we can omit the subscript " $i$." Suppose the sampling frequency is $2 W_{s}$ and the decision statistic of energy detection at each CR user is given by $\mathscr{R}=\left(1 / \sigma^{2}\right) \sum_{k=1}^{2 T_{s} W_{s}}|r(k)|^{2}$. It is shown in [26] that when $2 T_{s} W_{s}$ is large enough, according to central limit theorem, we have

$$
\mathscr{R} \sim \begin{cases}\mathcal{N}\left(2 T_{s} W_{s}, 2 T_{s} W_{s}\right), & \mathscr{H}_{0}, \\ \mathcal{N}\left(2 T_{s} W_{s}(1+\gamma), 2 T_{s} W_{s}(1+\gamma)^{2}\right), & \mathscr{H}_{1} .\end{cases}
$$

The probability density function (PDF) of $\mathscr{R}$ can then be written as

$$
\begin{gathered}
f_{\mathscr{R}}(r)=\frac{1}{2 \sqrt{\pi T_{s} W_{s}}} e^{-\left(r-2 T_{s} W_{s}\right)^{2} / 4 T_{s} W_{s}}, \quad \mathscr{H}_{0} . \\
f_{\mathscr{R}}(r)=\frac{1}{2(1+\gamma) \sqrt{\pi T_{s} W_{s}}} e^{-\left[r-2 T_{s} W_{s}(1+\gamma)\right]^{2} / 4 T_{s} W_{s}(1+\gamma)^{2}}, \\
\mathscr{H}_{1} .
\end{gathered}
$$

For a nonfading environment, the probability of false alarm and the probability of detection at each CR user can be computed by

$$
\begin{aligned}
p_{f} & =P\left\{\mathscr{R}>\lambda \mid \mathscr{H}_{0}\right\}=\int_{\lambda}^{\infty} f_{\mathscr{R} \mid \mathscr{H}_{0}}(r) d r \\
& =Q\left(\frac{\lambda}{\sqrt{2 T_{s} W_{s}}}-\sqrt{2 T_{s} W_{s}}\right), \\
p_{d} & =P\left\{\mathscr{R}>\lambda \mid \mathscr{H}_{1}\right\}=\int_{\lambda}^{\infty} f_{\mathscr{R} \mid \mathscr{H}_{1}}(r) d r \\
& =Q\left(\frac{\lambda}{(1+\gamma) \sqrt{2 T_{s} W_{s}}}-\sqrt{2 T_{s} W_{s}}\right),
\end{aligned}
$$

where $\lambda$ denotes the threshold of the energy detection and $Q(\cdot)$ is the $Q$-function defined as $Q(x)=(1 / \sqrt{2 \pi}) \int_{x}^{\infty} e^{-t^{2} / 2} d t$.
By (5), we have

$$
p_{f}=Q\left((1+\gamma) \cdot Q^{-1}\left(p_{d}\right)+\gamma \sqrt{2 T_{s} W_{s}}\right)
$$

In CSS, each CR user makes a "one bit" local decision (1 standing for the presence of PU and 0 standing for the absence of PU) on the primary user activity and then sends the individual decision to the fusion center over a reporting channel. Let $\Lambda$ denote the number of CR users reporting presence of PU. In the FC, the final decision strategy $\Phi(\cdot)$ is given by [27]

$$
\Phi= \begin{cases}\mathscr{H}_{0}, & \text { if } \Lambda<n \\ \mathscr{H}_{1}, & \text { if } \Lambda \geq n\end{cases}
$$

where $n$ is an integer, and $n=1,2, \ldots, K$ is the final decision threshold at FC. It is seen that OR rule corresponds to the case of $n=1$, AND rule corresponds to the case of $n=K$, and Majority rule corresponds to the case of $n=\lceil K / 2\rceil$. The final false alarm probability and final detection probability can be calculated as [27]

$$
\begin{aligned}
Q_{f} & =\sum_{i=n}^{K}\left(\begin{array}{c}
K \\
i
\end{array}\right) p_{f}^{i}\left(1-p_{f}\right)^{K-i}, \\
Q_{d} & =\sum_{i=n}^{K}\left(\begin{array}{c}
K \\
i
\end{array}\right) p_{d}^{i}\left(1-p_{d}\right)^{K-i} .
\end{aligned}
$$

Lets define $\mathscr{F}_{n}(x)=\sum_{i=n}^{K}\left(\begin{array}{c}K \\ i\end{array}\right) x^{i}(1-x)^{K-i}$ and $\mathscr{G}_{n}(x)=$ $d \mathscr{F}_{n}(x) / d x=K\left(\begin{array}{c}K-1 \\ n-1\end{array}\right) x^{n-1}(1-x)^{K-n}$. Then, we have $Q_{f}=$ $\mathscr{F}_{n}\left(p_{f}\right), Q_{d}=\mathscr{F}_{n}\left(p_{d}\right), d Q_{f} / d p_{f}=\mathscr{G}_{n}\left(p_{f}\right)$, and $d Q_{d} / d p_{d}=$ $\mathscr{G}_{n}\left(p_{d}\right)$.

\section{Optimization Problem Formulation}

In our proposed novel cooperative spectrum sensing frame structure, the CR user transmits data over the bandwidth $W_{t}$ only when the PU is sensed to be absent. Suppose that the power spectrum density of the CR user signal is evenly distributed, with the value of $S_{s}$. Then, $\sigma_{s}^{2}=S_{s} W_{t}$. There are two scenarios for the CR users to transmit data.

(1) The PU Is Correctly Detected to Be Absent. The probability of this scenario happening is $p\left(\mathscr{H}_{0}\right)\left(1-Q_{f}\right)$, where $p\left(\mathscr{H}_{0}\right)$ denotes the prior probability of the absence of the PU. In this case, the achievable throughput of the CR network is

$$
\mathbb{T}_{0}=W_{t} \log _{2}\left(1+\gamma_{s}\right) p\left(\mathscr{H}_{0}\right)\left(1-Q_{f}\right),
$$

where $\gamma_{s}$ is the SNR for the secondary link, and

$$
\gamma_{s}=\frac{\left|h_{s}\right|^{2} S_{s} W_{t}}{S_{n} W_{t}}
$$

where $h_{s}$ is the channel gain of the secondary link.

(2) The PU Is Falsely Detected to Be Absent. The probability of this scenario happening is $p\left(\mathscr{H}_{1}\right)\left(1-Q_{d}\right)$, where $p\left(\mathscr{H}_{1}\right)$ 
denotes the prior probability of the presence of the PU. In this case, the primary signal is considered as an interference to the secondary receiver, and the achievable throughput of the CR network is

$$
\mathbb{T}_{1}=W_{t} \log _{2}\left(1+\gamma_{S I}\right) p\left(\mathscr{H}_{1}\right)\left(1-Q_{d}\right),
$$

where $\gamma_{S I}$ is the signal-to-noise and interference ratio for the secondary link, and

$$
\gamma_{S I}=\frac{\left|h_{s}\right|^{2} S_{s} W_{t}}{\left(S_{p}\left|h_{p}\right|^{2}+S_{n}\right) W_{t}}=\frac{\gamma_{s}}{1+\gamma},
$$

where $h_{p}$ is the channel gain between the PU and the CR user.

Then, the achievable throughput of the CR network can be computed by

$$
\begin{aligned}
\mathbb{T}= & \mathbb{T}_{0}+\mathbb{T}_{1} \\
= & W_{t} \log _{2}\left(1+\gamma_{s}\right) p\left(\mathscr{H}_{0}\right)\left(1-Q_{f}\right) \\
& +W_{t} \log _{2}\left(1+\frac{\gamma_{s}}{1+\gamma}\right) p\left(\mathscr{H}_{1}\right)\left(1-Q_{d}\right) .
\end{aligned}
$$

Considering the fact that the priority of a CR system is the protection of the QoS of the primary link, a high probability of detection is required to ensure that no harmful interference is caused by the CR network. Our object is to find the optimal sensing settings to maximize the CR users' throughput under the condition of sufficient protection to PUs. To satisfy the required bandwidth for potential $\mathrm{CR}$ user data transmission, we set $W_{t}^{\text {th }} \leq W_{t}<W$; namely, $0<W_{s} \leq W-W_{t}^{\text {th }}$. Mathematically, the optimization problem can be stated as

$$
\begin{array}{ll}
\max & \mathbb{} \\
\text { s.t. } & Q_{d} \geq Q_{d}^{\text {th }}, \\
& 0<W_{s} \leq W-W_{t}^{\text {th }}, \\
& 1 \leq n \leq K,
\end{array}
$$

where $Q_{d}^{\text {th }}$ is the target detection probability with which the PUs are defined as being sufficiently protected. In the second scenario, the primary signal is considered as an interference with the secondary receiver, and we have $\log _{2}\left(1+\gamma_{s}\right)>$ $\log _{2}\left(1+\gamma_{S I}\right)$. Suppose that the prior probability of the presence of the PU is small; say less than 0.3; thus it is economically advisable to explore the secondary usage for PU spectrum band. Since $Q(x)$ is a decreasing function of $x$, according to (5), we have $p_{f}<p_{d}$. Furthermore, since $\mathscr{G}_{n}(x) \geq 0$ for $0 \leq$ $x \leq 1, \mathscr{F}_{n}(x)$ is an increasing function of $x$ for $0 \leq x \leq 1$; thus $\mathscr{F}_{n}\left(p_{f}\right)<\mathscr{F}_{n}\left(p_{d}\right)$ and $Q_{f}<Q_{d}$. Therefore, the optimization problem can be approximated by maximizing $\mathbb{T}_{0}$ subject to (16).

Theorem 1. $\mathbb{T}_{0}$ is a decreasing function of $Q_{d}$.

Proof. For a given $W_{s}$ and $n$, we have

$$
\frac{d \mathbb{T}_{0}}{d Q_{d}}=-p\left(\mathscr{H}_{0}\right)\left(W-W_{s}\right) \log _{2}\left(1+\gamma_{s}\right) \frac{d Q_{f}}{d Q_{d}},
$$

where

$$
\begin{gathered}
\frac{d Q_{f}}{d Q_{d}}=\frac{\left(d Q_{f}\right) /\left(d p_{d}\right)}{\left(d Q_{d}\right) /\left(d p_{d}\right)}=\frac{\mathscr{G}_{n}\left(p_{f}\right)}{\mathscr{G}_{n}\left(p_{d}\right)} \cdot \frac{d p_{f}}{d p_{d}}, \\
\frac{d p_{f}}{d p_{d}}=\frac{\left(d p_{f}\right) /(d \lambda)}{\left(d p_{d}\right) /(d \lambda)} \\
=(1+\gamma) \exp \left\{\frac{\lambda \gamma}{1+\gamma}-\frac{\lambda^{2} \gamma(\gamma+2)}{4 T_{s} W_{s}(1+\gamma)^{2}}\right\}>0 .
\end{gathered}
$$

Since $\mathscr{G}_{n}\left(p_{f}\right)>0$ and $\mathscr{G}_{n}\left(p_{d}\right)>0$, it is derived that $\left(d \mathbb{T}_{0}\right) /$ $d Q_{d}<0$. Thus, $\mathbb{T}_{0}$ is a decreasing function of $Q_{d}$. Theorem 1 is proved.

Therefore, the optimal solution must occur when the following equation stands:

$$
Q_{d}=\sum_{i=n}^{K}\left(\begin{array}{c}
K \\
i
\end{array}\right) p_{d}^{i}\left(1-p_{d}\right)^{K-i}=Q_{d}^{\text {th }}
$$

To get the optimal solution of (15), we need to calculate the root of $\Psi\left(p_{d}\right)=Q_{d}-Q_{d}^{\text {th }}=0$ numerically. Newton-Raphson algorithm can be employed to find the root of $\Psi\left(p_{d}\right)=Q_{d}-$ $Q_{d}^{\text {th }}=0$ [28]. The process of the Newton-Raphson algorithm is stated as follows.

(1) Choose tolerance $\delta$ and initial guess $p_{d, 1}$; let $j=1$.

(2) If $\left|\Psi\left(p_{d, j}\right)\right|<\delta$, stop; otherwise, go to step (3).

(3) Let $p_{d, j+1}=p_{d, j}-\Psi\left(p_{d, j}\right) / \mathscr{G}_{n}\left(p_{d, j}\right)$; let $j=j+1$; go to step (2).

The optimization problem is reduced to

$$
\begin{array}{ll}
\max & \mathbb{T}_{0} \\
\text { s.t. } & Q_{d}=Q_{d}^{\text {th }}, \\
& 0<W_{s} \leq W-W_{t}^{\text {th }}, \\
& 1 \leq n \leq K .
\end{array}
$$

In the next section, we will find the optimal solutions of $W_{s}$ and $n$ to maximize the throughput of the CR network.

\section{Optimal Sensing Settings of Throughput}

First we will prove that, for any target detection probability $Q_{d}^{\text {th }}$ and the final decision threshold $n$, there exists an optimal value of $W_{s}$ that maximizes the throughput of the CR network for the CSS with novel frame structure. The derivative of $\mathbb{T}_{0}$ with respect to $W_{s}$ is

$$
\begin{aligned}
\frac{d \mathbb{\mathbb { T }}_{0}}{d W_{s}}= & -p\left(\mathscr{H}_{0}\right)\left(1-Q_{f}\right) \log _{2}\left(1+\gamma_{s}\right) \\
& -p\left(\mathscr{H}_{0}\right) \frac{d Q_{f}}{d W_{s}}\left(W-W_{s}\right) \log _{2}\left(1+\gamma_{s}\right),
\end{aligned}
$$


where

$$
\begin{array}{r}
\frac{d Q_{f}}{d W_{s}}=\frac{d Q_{f}}{d p_{f}} \cdot \frac{d p_{f}}{d W_{s}}=\mathscr{G}_{n}\left(p_{f}\right) \frac{d p_{f}}{d W_{s}}, \\
\frac{d p_{f}}{d W_{s}}=-\frac{\gamma}{2} \sqrt{\frac{T_{s}}{\pi W_{s}}} \exp \left\{-\frac{1}{2}\left[(1+\gamma) \mathbb{Q}^{-1}\left(p_{d}\right)\right.\right. \\
\left.\left.+\gamma \sqrt{2 T_{s} W_{s}}\right]^{2}\right\} .
\end{array}
$$

Obviously,

$$
\begin{gathered}
\lim _{W_{s} \rightarrow 0} \frac{d \mathbb{T}_{0}}{d W_{s}}=\infty, \\
\lim _{W_{s} \rightarrow W} \frac{d \mathbb{T}_{0}}{d W_{s}}=-p\left(\mathscr{H}_{0}\right)\left(1-Q_{f}\right) \log _{2}\left(1+\gamma_{s}\right)<0 .
\end{gathered}
$$

Equation (24) means that $\mathbb{T}_{0}$ increases when $W_{s}$ is small and decreases when $W_{s}$ approaches $W$. Hence, there must be a maximum point of $\mathbb{T}_{0}$ for $W_{s} \in(0, W)$. Next we will prove that the maximum point of $\mathbb{T}_{0}$ is unique in this range; that is, there is a unique $W_{s}^{*}$ where $W_{s}^{*} \in(0, W)$ such that $d \mathbb{T}_{0} /\left.d W_{s}\right|_{W_{\mathrm{s}}=W_{s}^{*}}=0$. Setting $d \mathbb{T}_{0} / d W_{s}=0$ and after some algebraic manipulations, it is derived that $\mathscr{A}\left(W_{s}\right)=\mathscr{B}\left(W_{s}\right)$, where

$$
\begin{gathered}
\mathscr{A}\left(W_{s}\right)=-2 \ln \left[\frac{2(n-1) !(K-n) !}{\gamma K !} \sqrt{\frac{\pi}{T_{s}}} \frac{\sqrt{W_{s}}}{W-W_{s}}\right. \\
\left.\times \sum_{i=0}^{n-1}\left(\begin{array}{c}
K \\
i
\end{array}\right) p_{f}^{i-n+1}\left(1-p_{f}\right)^{n-i}\right], \\
\mathscr{B}\left(W_{s}\right)=\left[(1+\gamma) \mathscr{Q}^{-1}\left(p_{d}\right)+\gamma \sqrt{2 T_{s} W_{s}}\right]^{2} .
\end{gathered}
$$

If functions $\mathscr{A}\left(W_{s}\right)$ and $\mathscr{B}\left(W_{s}\right)$ intersect each other only once for $W_{s} \in(0, W)$, we can conclude that the root of $d \mathbb{T}_{0} / d W_{s}=0$ is unique. The derivative of $\mathscr{A}\left(W_{s}\right)$ with respect to $W_{s}$ is

$$
\begin{aligned}
\frac{d \mathscr{A}\left(W_{s}\right)}{d W_{s}}= & -\frac{W+W_{s}}{W_{s}\left(W-W_{s}\right)}+\frac{2}{\sum_{i=0}^{n-1}\left(\begin{array}{l}
K \\
i
\end{array}\right) p_{f}^{i-n+1}\left(1-p_{f}\right)^{n-i}} \\
& \times \sum_{i=0}^{n-1}\left(\begin{array}{c}
K \\
i
\end{array}\right)\left[(n-1-i) p_{f}^{i-n}\left(1-p_{f}\right)^{n-i}\right. \\
& \left.+(n-i) p_{f}^{i-n+1}\left(1-p_{f}\right)^{n-i-1}\right] \frac{d p_{f}}{d W_{s}} .
\end{aligned}
$$

Since $n-1-i \geq 0$ and $n-i>0$ for $i=0,1, \ldots, n-1$, and $d p_{f} / d W_{s}<0$, we can obtain that $d \mathscr{A}\left(W_{s}\right) / d W_{s}<0$ for $W_{s} \in(0, W)$. Thus, $\mathscr{A}\left(W_{s}\right)$ is a decreasing function of $W_{s}$ for $W_{s} \in(0, W)$. The derivative of $\mathscr{B}\left(W_{s}\right)$ with respect to $W_{s}$ is

$$
\frac{d \mathscr{B}\left(W_{s}\right)}{d W_{s}}=\gamma \sqrt{\frac{2 T_{s}}{W_{s}}}\left[(1+\gamma) \mathscr{Q}^{-1}\left(p_{d}\right)+\gamma \sqrt{2 T_{s} W_{s}}\right] .
$$

Case 1. If $\mathbb{Q}^{-1}\left(p_{d}\right) \geq 0$, we have $(1+\gamma) \mathbb{Q}^{-1}\left(p_{d}\right)+\gamma \sqrt{2 T_{s} W_{s}}>$ 0 for $W_{s} \in(0, W)$. According to (27), we can obtain that $d \mathscr{B}\left(W_{s}\right) / d W_{s}>0$. In this case, $\mathscr{B}\left(W_{s}\right)$ is an increasing function of $W_{s}$ for $W_{s} \in(0, W)$. Thus, $\mathscr{A}\left(W_{s}\right)$ and $\mathscr{B}\left(W_{s}\right)$ can intersect at most once. Therefore, there is only one intersection between $\mathscr{A}\left(W_{s}\right)$ and $\mathscr{B}\left(W_{s}\right)$ for $W_{s} \in(0, W)$. The root of $d \mathbb{T}_{0} / d W_{s}=0$ is unique in this case.

Case 2. If $\mathscr{Q}^{-1}\left(p_{d}\right)<0$, when $0<W_{s}<\left[(1+\gamma) \mathscr{Q}^{-1}\left(p_{d}\right)\right]^{2} /$ $2 T_{s} \gamma^{2}$, we have $(1+\gamma) \mathscr{Q}^{-1}\left(p_{d}\right)+\gamma \sqrt{2 T_{s} W_{s}}<0$ and $d \mathscr{B}\left(W_{s}\right) /$ $d W_{s}<0$. Thus, $\mathscr{B}\left(W_{s}\right)$ is a decreasing function of $W_{s}$ for $W_{s} \in\left(0,\left[(1+\gamma) \mathscr{Q}^{-1}\left(p_{d}\right)\right]^{2} / 2 T_{s} \gamma^{2}\right)$. When $\left[(1+\gamma) \mathscr{Q}^{-1}\left(p_{d}\right)\right]^{2} /$ $2 T_{s} \gamma^{2} \leq W_{s}<W$, we have $(1+\gamma) Q^{-1}\left(p_{d}\right)+\gamma \sqrt{2 T_{s} W_{s}} \geq 0$ and $d \mathscr{B}\left(W_{s}\right) / d W_{s} \geq 0$. Thus, $\mathscr{B}\left(W_{s}\right)$ is an increasing function of $W_{s}$ for $W_{s} \in\left[\left[(1+\gamma) Q^{-1}\left(p_{d}\right)\right]^{2} / 2 T_{s} \gamma^{2}, W\right)$. In this case, we will prove that $\mathscr{A}\left(W_{s}\right)$ and $\mathscr{B}\left(W_{s}\right)$ can intersect each other only once for $W_{s} \in(0, W)$.

Theorem 2. $d \mathscr{A}\left(W_{s}\right) / d W_{s}<d \mathscr{B}\left(W_{s}\right) / d W_{s}$ for $W_{s} \in(0, W)$.

Proof. For $W_{s} \in\left[\left[(1+\gamma) \mathbb{Q}^{-1}\left(p_{d}\right)\right]^{2} / 2 T_{s} \gamma^{2}, W\right)$, we have $d \mathscr{A}$ $\left(W_{s}\right) / d W_{s}<0$ and $d \mathscr{B}\left(W_{s}\right) / d W_{s} \geq 0$. Thus, $d \mathscr{A}\left(W_{s}\right) / d W_{s}<$ $d \mathscr{B}\left(W_{s}\right) / d W_{s}$.

For $W_{s} \in\left(0,\left[(1+\gamma) \mathscr{Q}^{-1}\left(p_{d}\right)\right]^{2} / 2 T_{s} \gamma^{2}\right)$, we have $d \mathscr{A}\left(W_{s}\right) /$ $d W_{s}<0$ and $d \mathscr{B}\left(W_{s}\right) / d W_{s}<0$. We first prove that, when $n=$ 1, $d \mathscr{A}\left(W_{s}\right) / d W_{s}<d \mathscr{B}\left(W_{s}\right) / d W_{s}$. According to (26), when $n=1$, we have

$$
\left.\frac{d \mathscr{A}\left(W_{s}\right)}{d W_{s}}\right|_{n=1}=-\frac{W+W_{s}}{W_{s}\left(W-W_{s}\right)}+\frac{2}{1-p_{f}} \cdot \frac{d p_{f}}{d W_{s}} .
$$

According to (27) and (28), $d \mathscr{A}\left(W_{s}\right) /\left.d W_{s}\right|_{n=1}<d \mathscr{B}\left(W_{s}\right)$ $/ d W_{s}$ is given as

$$
\begin{aligned}
& -\frac{W+W_{s}}{W_{s}\left(W-W_{s}\right)} \\
& -\frac{\gamma}{1-\mathscr{Q}\left((1+\gamma) \cdot Q^{-1}\left(p_{d}\right)+\gamma \sqrt{2 T_{s} W_{s}}\right)} \sqrt{\frac{T_{s}}{\pi W_{s}}} \\
& \times \exp \left\{-\frac{1}{2}\left[(1+\gamma) Q^{-1}\left(p_{d}\right)+\gamma \sqrt{2 T_{s} W_{s}}\right]^{2}\right\} \\
& <\gamma \sqrt{\frac{2 T_{s}}{W_{s}}}\left[(1+\gamma) \mathbb{Q}^{-1}\left(p_{d}\right)+\gamma \sqrt{2 T_{s} W_{s}}\right] .
\end{aligned}
$$

For $W_{s} \in\left(0,\left[(1+\gamma) \mathbb{Q}^{-1}\left(p_{d}\right)\right]^{2} / 2 T_{s} \gamma^{2}\right),(1+\gamma) \mathbb{Q}^{-1}\left(p_{d}\right)+$ $\gamma \sqrt{2 T_{s} W_{s}}<0$. Let $x=-\left[(1+\gamma) \mathscr{Q}^{-1}\left(p_{d}\right)+\gamma \sqrt{2 T_{s} W_{s}}\right]>0$. Substituting $x$ into (29), it is derived that

$$
\frac{W+W_{s}}{\gamma\left(W-W_{s}\right){\sqrt{2 T_{s} W_{s}}}}+\frac{1}{\sqrt{2 \pi} Q(x)} e^{-x^{2} / 2}>x .
$$

Since $(1 / \sqrt{2 \pi} Q(x)) e^{-x^{2} / 2}>x$ for $x \geq 0$ [29], the inequality at (30) is verified. Thus, $d \mathscr{A}\left(W_{s}\right) /\left.d W_{s}\right|_{n=1}<d \mathscr{B}\left(W_{s}\right) /$ $d W_{s}$ has been proved. Next we will prove that $d \mathscr{A}\left(W_{s}\right) / d W_{s}<$ 
$d \mathscr{B}\left(W_{s}\right) / d W_{s}$ for $n=2,3, \ldots, K$. Since $n-i \geq 1$ for $i=$ $0,1, \ldots, n-1$, we have

$$
\begin{aligned}
& \frac{2}{\sum_{i=0}^{n-1}\left(\begin{array}{c}
K \\
i
\end{array}\right) p_{f}^{i-n+1}\left(1-p_{f}\right)^{n-i}} \\
& \quad \times \sum_{i=0}^{n-1}\left(\begin{array}{c}
K \\
i
\end{array}\right)\left[(n-1-i) p_{f}^{i-n}\left(1-p_{f}\right)^{n-i}\right. \\
& \left.+(n-i) p_{f}^{i-n+1}\left(1-p_{f}\right)^{n-i-1}\right] \\
& >2 \frac{\sum_{i=0}^{n-1}\left(\begin{array}{c}
K \\
i
\end{array}\right)(n-i) p_{f}^{i-n+1}\left(1-p_{f}\right)^{n-i-1}}{\sum_{i=0}^{n-1}\left(\begin{array}{c}
K \\
i
\end{array}\right) p_{f}^{i-n+1}\left(1-p_{f}\right)^{n-i-1}} \cdot \frac{1}{1-p_{f}} \\
& >\frac{2}{1-p_{f}}
\end{aligned}
$$

According to (26), (28), and (31), we obtain $d \mathscr{A}\left(W_{s}\right) /\left.d W_{s}\right|_{n=2,3, \ldots, K}<d \mathscr{A}\left(W_{s}\right) /\left.d W_{s}\right|_{n=1}$. Therefore, $d \mathscr{A}$ $\left(W_{s}\right) / d W_{s}<d \mathscr{B}\left(W_{s}\right) / d W_{s}$ for $W_{s} \in(0, W)$. Theorem 2 is proved.

In Case 2, there are two possible scenarios for the intersection between $\mathscr{A}\left(W_{s}\right)$ and $\mathscr{B}\left(W_{s}\right)$.

(i) $\mathscr{A}\left(W_{s}\right)$ and $\mathscr{B}\left(W_{s}\right)$ intersect each other in the region of $W_{s} \in\left(0,\left[(1+\gamma) Q^{-1}\left(p_{d}\right)\right]^{2} / 2 T_{s} \gamma^{2}\right)$. Since $d \mathscr{A}\left(W_{s}\right) / d W_{s}<d \mathscr{B}\left(W_{s}\right) / d W_{s}$, it is impossible for them to intersect more than once because $\mathscr{A}\left(W_{s}\right)$ decreases at a faster rate than $\mathscr{B}\left(W_{s}\right)$ for $W_{s} \in\left(0,\left[(1+\gamma) Q^{-1}\left(p_{d}\right)\right]^{2} / 2 T_{s} \gamma^{2}\right)$. For $W_{s} \in$ $\left[\left[(1+\gamma) \mathscr{Q}^{-1}\left(p_{d}\right)\right]^{2} / 2 T_{s} \gamma^{2}, W\right), \mathscr{B}\left(W_{s}\right)$ is always larger than $\mathscr{A}\left(W_{s}\right)$ since $\mathscr{B}\left(W_{s}\right)$ is an increasing function of $W_{s}$ while $\mathscr{A}\left(W_{s}\right)$ is a decreasing function of $W_{s}$. It is impossible for them to intersect each other for $W_{s} \in\left[\left[(1+\gamma) Q^{-1}\left(p_{d}\right)\right]^{2} / 2 T_{s} \gamma^{2}, W\right)$. Therefore, in this scenario, there is only one intersection between $\mathscr{A}\left(W_{s}\right)$ and $\mathscr{B}\left(W_{s}\right)$, and it occurs in the region of $W_{s} \in\left(0,\left[(1+\gamma) Q^{-1}\left(p_{d}\right)\right]^{2} / 2 T_{s} \gamma^{2}\right)$.

(ii) $\mathscr{A}\left(W_{s}\right)$ and $\mathscr{B}\left(W_{s}\right)$ do not intersect each other in the region of $W_{s} \in\left(0,\left[(1+\gamma) Q^{-1}\left(p_{d}\right)\right]^{2} / 2 T_{s} \gamma^{2}\right)$. In this scenario, they must intersect in the region of $W_{s} \in\left[\left[(1+\gamma) Q^{-1}\left(p_{d}\right)\right]^{2} / 2 T_{s} \gamma^{2}, W\right)$ since there must be at least one intersection in the entire range of $0<$ $W_{s}<W$. For $W_{s} \in\left[\left[(1+\gamma) Q^{-1}\left(p_{d}\right)\right]^{2} / 2 T_{s} \gamma^{2}, W\right)$, $\mathscr{A}\left(W_{s}\right)$ is a decreasing function of $W_{s}$ and $\mathscr{B}\left(W_{s}\right)$ is an increasing function of $W_{s}$. Thus, they can intersect each other at most once. Therefore, in this scenario, there is only one intersection between $\mathscr{A}\left(W_{s}\right)$ and $\mathscr{B}\left(W_{s}\right)$, and it occurs in the region of $W_{s} \in$ $\left[\left[(1+\gamma) Q^{-1}\left(p_{d}\right)\right]^{2} / 2 T_{s} \gamma^{2}, W\right)$.

From the analysis above, one can conclude that there is a unique $W_{s}^{*}$ where $W_{s}^{*} \in(0, W)$ such that $d \mathbb{T}_{0} /\left.d W_{s}\right|_{W_{s}=W_{s}^{*}}=$ 0 . Bisection method can be used to find the root of $d \mathbb{T}_{0} / d W_{s}=$ 0 [28]. The process of bisection method is stated as follows.
(1) Choose lower guess $W_{s}^{l}$ and upper guess $W_{s}^{u}$ for the root such that the function changes sign over the interval. This can be checked by ensuring that $d \mathbb{\mathbb { V }}_{0} /\left.d W_{s}\right|_{W_{s}=W_{s}^{l}} \cdot d \mathbb{T}_{0} /\left.d W_{s}\right|_{W_{s}=W_{s}^{u}}<0$.

(2) An estimate of the root $W_{s}^{r}$ is determined by $W_{s}^{r}=$ $\left(W_{s}^{l}+W_{s}^{u}\right) / 2$.

(3) Make the following evaluations to determine in which subinterval the root lies.

(a) If $d \mathbb{\mathbb { T }}_{0} /\left.d W_{s}\right|_{W_{s}=W_{s}^{l}} \cdot d \mathbb{\mathbb { T }}_{0} /\left.d W_{s}\right|_{W_{s}=W_{s}^{r}}<0$, the root lies in the lower subinterval. Therefore, set $W_{s}^{u}=$ $W_{s}^{r}$ and return to step (2).

(b) If $d \mathbb{\mathbb { T }}_{0} /\left.d W_{s}\right|_{W_{s}=W_{s}^{l}} \cdot d \mathbb{\mathbb { T }}_{0} /\left.d W_{s}\right|_{W_{s}=W_{s}^{r}}>0$, the root lies in the upper subinterval. Therefore, set $W_{s}^{l}=$ $W_{s}^{r}$ and return to step (2).

(c) If $d \mathbb{\mathbb { T }}_{0} /\left.d W_{s}\right|_{W_{s}=W_{s}^{l}} \cdot d \mathbb{\mathbb { T }}_{0} /\left.d W_{s}\right|_{W_{s}=W_{s}^{r}}=0$, the root equals $W_{s}^{r}$; terminate the computation.

Note that the second constraint of the optimization problem is $0<W_{s} \leq W-W_{t}^{\text {th }}$. If $W_{s}^{*} \geq W-W_{t}^{\text {th }}, \mathbb{T}_{0}$ is monotonically increasing in the range of $0<W_{s} \leq W-W_{t}^{\text {th }}$. In this case, choose $W_{s}=W-W_{t}^{\text {th }}$; the optimization is achieved. If $0<W_{s}^{*}<W-W_{t}^{\text {th }}, \mathbb{T}_{0}$ is monotonically increasing in the range of $0<W_{s} \leq W_{s}^{*}$ and is monotonically decreasing in the range of $W_{s}^{*}<W_{s}<W-W_{t}^{\text {th }}$. In this case, choose $W_{s}=W_{s}^{*}$; the optimization is achieved.

For the third constraint of the optimization problem, $1 \leq$ $n \leq K$, no closed-form solution for the optimal $n^{*}$ is available. However, since $n$ is an integer, it is not computationally expensive to search through $n$ from 1 to $K$ to obtain the optimal $n^{*}$ that maximizes (20). Therefore, the process to achieve the maximum throughput for the CSS with novel frame structure is listed as follows.

(1) For each $n(1 \leq n \leq K)$, calculate the root $p_{d n}$ of $\Psi\left(p_{d \_n}\right)=0$ by using the Newton-Raphson algorithm.

(2) For each $p_{d n n}$, calculate the root $W_{s, n}^{*}$ of $d \mathbb{T}_{0} / d W_{s}=0$ by using the bisection method. If $W_{s \_n}^{*} \geq W-W_{t}^{\text {th }}$ is satisfied, choose $W_{s \_n}^{\mathrm{opt}}=W-W_{t}^{\mathrm{th}}$; otherwise, choose $W_{s\lrcorner n}^{\mathrm{opt}}=W_{s \_}^{*}$.

(3) For each $W_{s_{-} n}^{\mathrm{opt}}$, calculate the corresponding individual false alarm probability $p_{f \_}$by (6), the final false alarm probability $Q_{f \_n}$ by $(8)$, and the throughput $\mathbb{T}_{0 \_n}$ by (10).

(4) Compare $\mathbb{T}_{0 \_}$and choose the maximum one.

\section{Simulation Results}

To get insight into the effectiveness of the proposed sensing methods and validate some related theorems, computer simulations have been conducted to evaluate the performance of throughput for various spectrum sensing schemes. In the simulations, we set the bandwidth of PU as $W=20000 \mathrm{~Hz}$; the frame duration is $T=20 \mathrm{~ms}$; the individual reporting duration is $T_{r}=1 \mathrm{~ms}$; the target detection probability is 


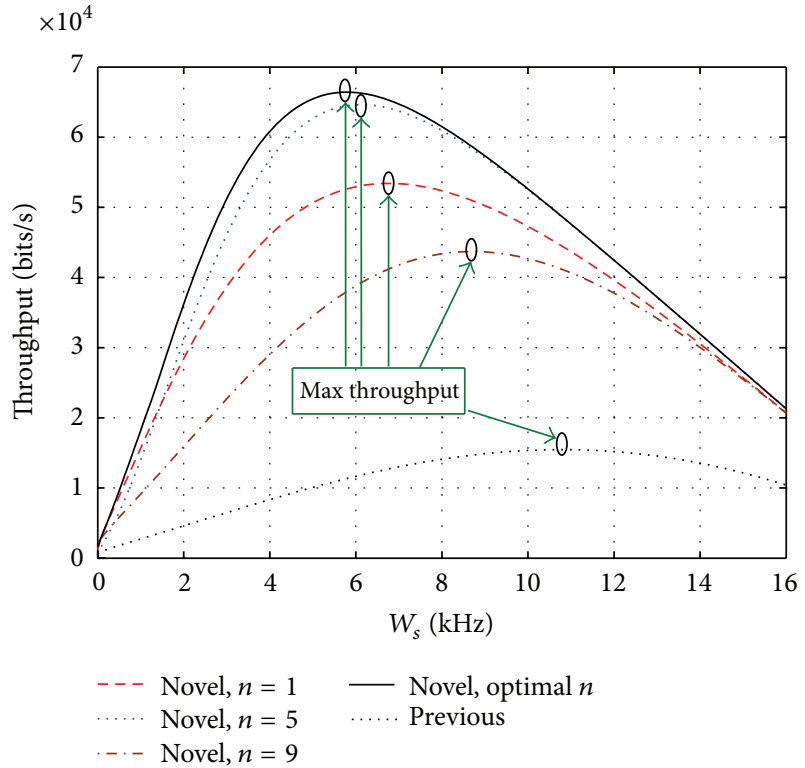

FIGURE 2: The throughput of the CR network versus the sensing bandwidth $W_{s}$ for various sensing schemes.

$Q_{d}^{\text {th }}=99 \%$; the SNR for the secondary link is $\gamma_{s}=20 \mathrm{~dB}$; the prior probability of the absence of $\mathrm{PU}$ is $p\left(\mathscr{H}_{0}\right)=0.8$ and $p\left(\mathscr{H}_{1}\right)=1-p\left(\mathscr{H}_{0}\right)=0.2$. To satisfy the required bandwidth for potential CR user data transmission, we set $W_{t}^{\text {th }}=W / 5$.

Figure 2 compares the throughput of the CR network when the novel CSS frame structure is employed to the case when the previous frame structure is employed. For the previous frame structure, we can refer to Figure 1 in [25]; for the novel CSS scheme, the number of CR users is $K=9$; the SNR of the PU's signal at the CR user is $\gamma=-10 \mathrm{~dB}$. It is seen that using the novel CSS frame structure can achieve a much higher throughput than that using the previous frame structure, especially when the final decision threshold $n$ is optimized. For the previous frame structure, the maximum achievable throughput is approximately $1.55 \times 10^{4} \mathrm{bits} / \mathrm{s}$. However, when our proposed novel CSS frame structure is employed and $n$ is optimized, the maximum achievable throughput is approximately $6.64 \times 10^{4} \mathrm{bits} / \mathrm{s}$. From this figure, it can be seen that, for a given sensing bandwidth, the optimal sensing settings improve the throughput of the CR network. When $W_{s} \geq 1.6 \mathrm{kHz}$, Majority rule $(n=5)$ is suboptimal. However, OR rule $(n=1)$ outperforms Majority rule when $0<W_{s}<1.6 \mathrm{kHz}$. It is also observed that there exists an optimal sensing bandwidth which yields the highest throughput for the secondary network. The optimal sensing bandwidth varies with different values of $n$.

In Figure 3, it is also observed that the throughput of the novel CSS scheme is much higher than that of the previous scheme. For the novel CSS scheme, optimal $n$ values are used for each fixed $W_{s}$. The optimal sensing settings can achieve a higher throughput than that using fixed sensing bandwidth. In addition, one can clearly see that the disadvantage with a fixed sensing bandwidth is that, at high SNR levels where the PU can be easily detected, the throughput of the CR network

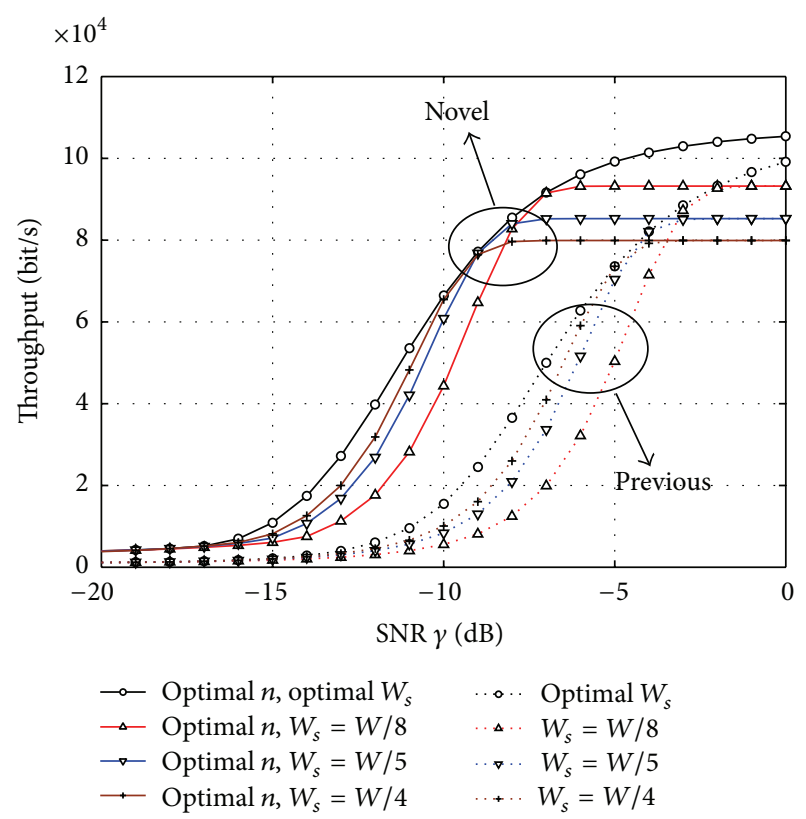

FIGURE 3: The throughput of the CR network versus SNR for various values of $W_{s}$; optimal $n$ values are used for each fixed $W_{s}$ in the novel CSS scheme.

is bounded by the percentage of the total PU transmission bandwidth that is assigned to spectrum sensing. For the novel CSS scheme, by comparing the curves of $W_{s}=W / 5$ and $W_{s}=$ $W / 4$, we can see that, in low SNR region, $-20 \sim-9 \mathrm{~dB}$, the throughput of the former scheme $\left(W_{s}=W / 5\right)$ is lower than that of the latter scheme $\left(W_{s}=W / 4\right)$. However, when SNR is larger than $-9 \mathrm{~dB}$, the two curves approach constants and the throughput of the former scheme is larger than that of the latter scheme. This indicates that no fixed $W_{s}$ is optimal for all SNR values. Thus, there is a need to optimize the value of $W_{s}$ to enhance the throughput of the CR network.

Figure 4 illustrates the maximum throughput of the $\mathrm{CR}$ network versus the average SNR for the novel CSS scheme with various counting rules. Optimal sensing bandwidth is used in each of the counting rules. One can see clearly that the optimal sensing settings can achieve a higher throughput than that using uniform thresholds. It is seen that, when $-15 \mathrm{~dB} \leq \mathrm{SNR} \leq 0 \mathrm{~dB}$, Majority rule $(n=5)$ is suboptimal and AND rule $(n=9)$ performs the worst. In particular, at $-10 \mathrm{~dB}$ SNR, the optimal sensing settings can achieve almost 1.5 times throughput than that when AND rule is used. However, when SNR is lower than $-15 \mathrm{~dB}$, AND rule outperforms Majority rule and OR rule $(n=1)$. And it achieves the same throughput as the optimal setting when SNR is extremely weak. Therefore, there is no single decision threshold $n$ that is optimal for all cases. To maximize the throughput of the CR network, different SNR levels require different optimal $n$ values.

Figure 5 is simulated to show the maximum throughput of the CR network versus number of CR users $K$ for various sensing schemes with different values of $W_{s}$, in which optimal $n$ values are used for each fixed $W_{s}$. The SNR of PU's signal 


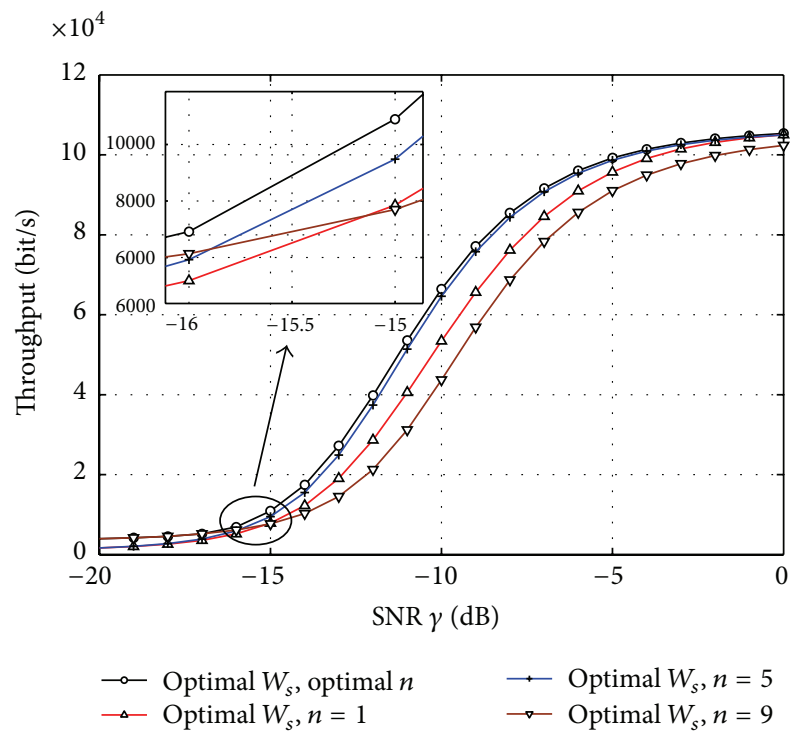

FIGURE 4: The maximum throughput versus SNR for various counting rules; optimal sensing bandwidth is used in each of the counting rules.

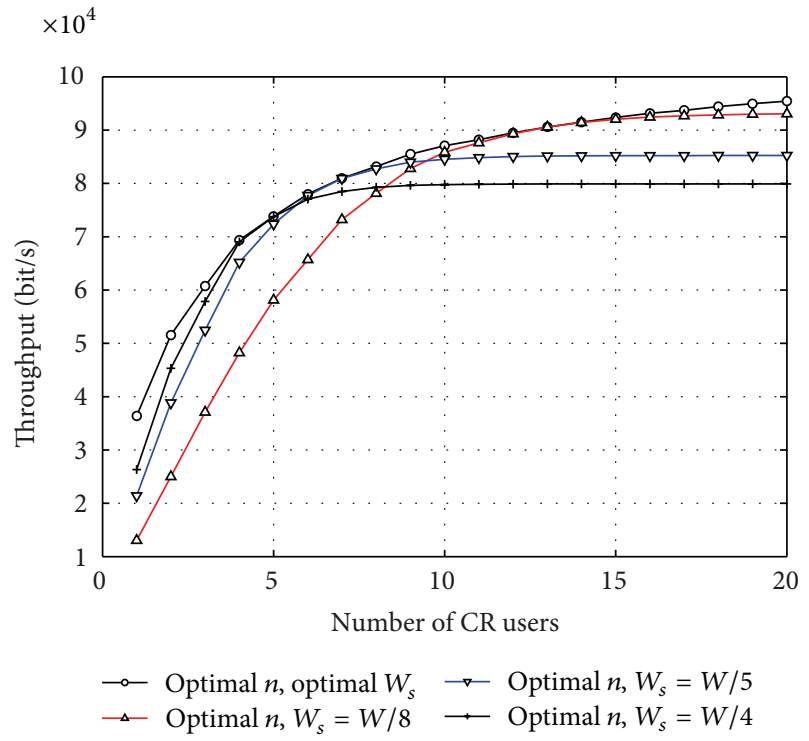

FIGURE 5: The maximum throughput versus the number of CR users for various values of $W_{s}$; optimal $n$ values are used for each fixed $W_{s}$.

at the CR user is $\gamma=-8 \mathrm{~dB}$. It has been shown that the throughput with optimal sensing settings is larger than that using fixed sensing bandwidth and grows as the number of $\mathrm{CR}$ users increases. For the sensing scheme with fixed sensing bandwidth, the throughput of the CR network approaches constants when the number of CR users is extremely large and is bounded by the percentage of the total PU transmission bandwidth that is assigned to spectrum sensing. By comparing the curves of the sensing schemes with different values of $W_{s}$, we can also find that no fixed $W_{s}$ is optimal for all cases. To enhance the throughput of the CR network, the value of $W_{s}$ needs to be optimized.

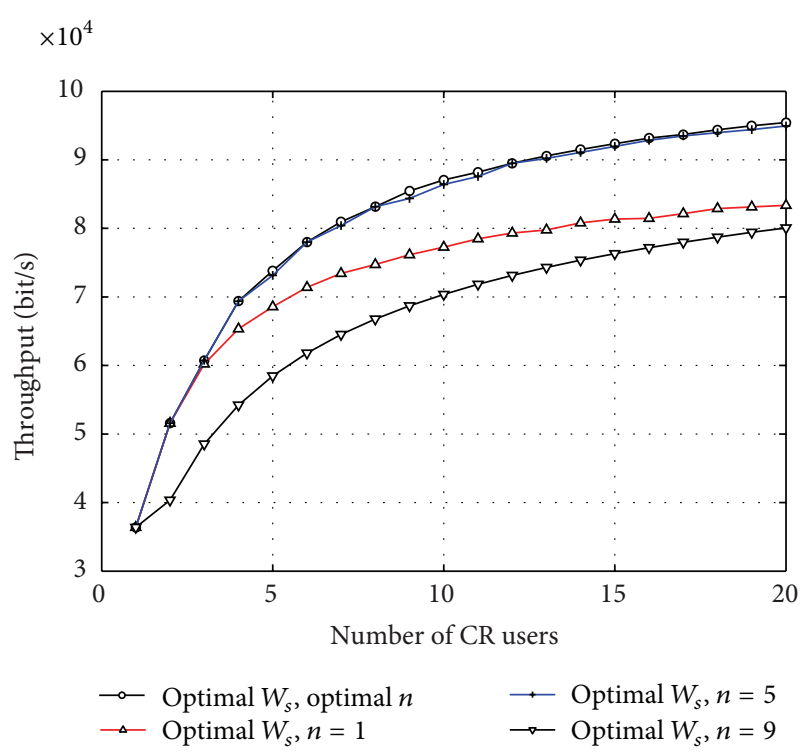

FIGURE 6: The maximum throughput versus the number of CR users for various counting rules; optimal sensing bandwidth is used in each of the counting rules.

In Figure 6, the maximum throughput of the CR network is presented versus the number of CR users $K$ for the novel CSS scheme with various counting rules. Optimal sensing bandwidth is used in each of the counting rules. The SNR of PU's signal at the CR user is $\gamma=-8 \mathrm{~dB}$. It is observed that the optimal sensing settings can achieve a higher throughput than that using uniform thresholds. Majority rule $(n=5)$ is suboptimal and AND rule $(n=9)$ always performs the worst. It is also seen that the throughput of the CR network increases as the number of CR users increases. However, the complexity of our proposed algorithm grows approximately linearly with the number of cooperating CR users.

\section{Conclusions}

In this paper, we propose a novel frame structure for cooperative spectrum sensing. It has been proved that there exists an optimal sensing bandwidth which yields the highest throughput for the CR network. The optimal sensing settings to maximize the throughput of the $\mathrm{CR}$ network under the conditions of sufficient protection to PUs and required bandwidth for potential CR user data transmission have been proposed. The proposed optimal sensing settings are analyzed and calculated in detail by using some simple but reliable methods. Computer simulations have shown that significant improvement in the throughput of the CR network has been achieved when the sensing bandwidth and the final decision threshold are jointly optimized.

\section{Conflict of Interests}

The authors declare that there is no conflict of interests regarding the publication of this paper. 


\section{Acknowledgments}

This work is supported by the National Fundament Research of China (973 no. 2009CB3020400) and the Jiangsu Province Natural Science Foundation under Grant BK2011002.

\section{References}

[1] P. P. Chen and Q. Y. Zhang, "Joint temporal and spatial sensing based cooperative cognitive networks," IEEE Communications Letters, vol. 15, no. 5, pp. 530-532, 2011.

[2] Y.-J. Choi, Y. Xin, and S. Rangarajan, "Overhead-throughput tradeoff in cooperative cognitive radio networks," in Proceedings of the IEEE Wireless Communications and Networking Conference (WCNC '09), pp. 1-6, April 2009.

[3] D. Cabric, S. M. Mishra, and R. W. Brodersen, "Implementation issues in spectrum sensing for cognitive radios," in Conference Record of the 38th Asilomar Conference on Signals, Systems and Computers, pp. 772-776, November 2004.

[4] Z. Quan, S. Cui, and A. H. Sayed, "Optimal linear cooperation for spectrum sensing in cognitive radio networks," IEEE Journal on Selected Topics in Signal Processing, vol. 2, no. 1, pp. 28-40, 2008.

[5] Z. Quan, S. Cui, A. H. Sayed, and H. V. Poor, "Optimal multiband joint detection for spectrum sensing in cognitive radio networks," IEEE Transactions on Signal Processing, vol. 57, no. 3, pp. 1128-1140, 2009.

[6] G. Ganesan, Y. Li, B. Bing, and S. Li, "Spatiotemporal sensing in cognitive radio networks," IEEE Journal on Selected Areas in Communications, vol. 26, no. 1, pp. 5-12, 2008.

[7] J. Jafarian and K. H. Hamdi, "Throughput optimization in a cooperative double-threshold sensing scheme," in Proceedings of the IEEE Wireless Communications and Networking Conference, pp. 1034-1038, 2012.

[8] J. Jafarian and K. H. Hamdi, "Non-cooperative doublethreshold sensing scheme: a sensing-throughput tradeoff," in Proceedings of the IEEE Wireless Communications and Networking Conference, pp. 3405-3410, 2013.

[9] G. Ganesan and Y. Li, "Cooperative spectrum sensing in cognitive radio, part I: two user networks," IEEE Transactions on Wireless Communications, vol. 6, no. 6, pp. 2204-2212, 2007.

[10] G. Ganesan and Y. Li, "Cooperative spectrum sensing in cognitive radio, part II: multiuser networks," IEEE Transactions on Wireless Communications, vol. 6, no. 6, pp. 2214-2222, 2007.

[11] Q. Wu, G. Ding, J. Wang, and Y.-D. Yao, "Spatialtemporal opportunity detection for spectrum-heterogeneous cognitive radio networks: two-dimensional sensing," IEEE Transactions on Wireless Communications, vol. 12, pp. 516-526, 2013.

[12] G. Ding, J. Wang, Q. Wu, F. Song, and Y. Chen, "Spectrum sensing in opportunity-heterogeneous cognitive sensor networks: how to cooperate?" IEEE Sensors Journal, vol. 13, pp. 4247-4255, 2013.

[13] M. Perc, J. Gómez-Gardeñes, A. Szolnoki, L. M. Floría, and Y. Moreno, "Evolutionary dynamics of group interactions on structured populations: a review," Journal of the Royal Society Interface, vol. 10, no. 80, Article ID 20120997, 2013.

[14] M. Perc and P. Grigolini, "Collective behavior and evolutionary games-an introduction," Chaos, Solitons \& Fractals, vol. 56, pp. $1-5,2013$.

[15] A. Szolnoki, M. Perc, and G. Szabó, "Accuracy in strategy imitations promotes the evolution of fairness in the spatial ultimatum game," Europhysics Letters, vol. 100, no. 2, Article ID 28005, 2012.

[16] A. Szolnoki, M. Perc, and G. Szabó, "Defense mechanisms of empathetic players in the spatial ultimatum game," Physical Review Letters, vol. 109, Article ID 078701, 2012.

[17] M. Perc and A. Szolnoki, "Coevolutionary games-A mini review," BioSystems, vol. 99, no. 2, pp. 109-125, 2010.

[18] Y. Xu, J. Wang, Q. Wu, A. Anpalagan, and Y.-D. Yao, “Opportunistic spectrum access in cognitive radio networks: global optimization using local interaction games," IEEE Journal on Selected Topics in Signal Processing, vol. 6, no. 2, pp. 180-194, 2012.

[19] W. Zhong, Y. Xu, and H. Tianfield, "Game-theoretic opportunistic spectrum sharing strategy selection for cognitive MIMO multiple access channels," IEEE Transactions on Signal Processing, vol. 59, no. 6, pp. 2745-2759, 2011.

[20] B. Wang, K. J. Ray Liu, and T. Charles Clancy, "Evolutionary cooperative spectrum sensing game: how to collaborate?" IEEE Transactions on Communications, vol. 58, pp. 890-900, 2010.

[21] H. Vu-Van and I. Koo, "Cooperative spectrum sensing with collaborative users using individual sensing credibility for cognitive radio network," IEEE Transactions on Consumer Electronics, vol. 57, no. 2, pp. 320-326, 2011.

[22] S. Stotas and A. Nallanathan, "On the throughput and spectrum sensing enhancement of opportunistic spectrum access cognitive radio networks," IEEE Transactions on Wireless Communications, vol. 11, no. 1, pp. 97-107, 2012.

[23] H. Hu, H. Zhang, and H. Yu, "Delay QoS guaranteed cooperative spectrum sensing in cognitive radio networks," International Journal of Electronics and Communications, vol. 67, pp. 804-807, 2013.

[24] H. Yu, W. Tang, and S. Li, "Optimization of cooperative spectrum sensing with sensing user selection in cognitive radio networks," EURASIP Journal on Wireless Communications and Networking, vol. 208, article 208, 2011.

[25] W. Zhang, R. K. Mallik, and K. Ben Letaief, “Optimization of cooperative spectrum sensing with energy detection in cognitive radio networks," IEEE Transactions on Wireless Communications, vol. 8, no. 12, pp. 5761-5766, 2009.

[26] W. Yin, P. Ren, Q. Du, and Y. Wang, "Delay and throughput oriented continuous spectrum sensing schemes in cognitive radio networks," IEEE Transactions on Wireless Communications, vol. 11, no. 6, pp. 2148-2159, 2012.

[27] W. Han, J. Li, Z. Tian, and Y. Zhang, "Efficient cooperative spectrum sensing with minimum overhead in cognitive radio," IEEE Transactions on Wireless Communications, vol. 9, no. 10, pp. 3006-3011, 2010.

[28] C. Steven Chapra and P. Raymond Canale, Numerical Methods for Engineers, McGraw-Hill, 6th edition, 2010.

[29] N. Kingsbury, "Approximation Formulae for the Gaussian Error Integral, Q(x)," June 2005, Connexions, http://cnx.org/ content $/ \mathrm{m} 11067 / 2.4 /$. 


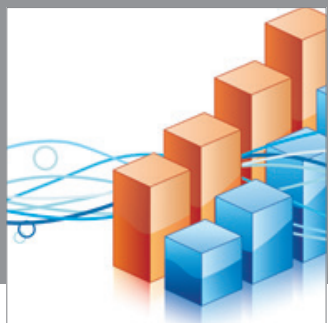

Advances in

Operations Research

mansans

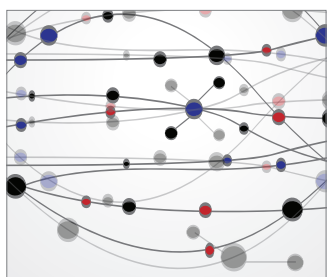

The Scientific World Journal
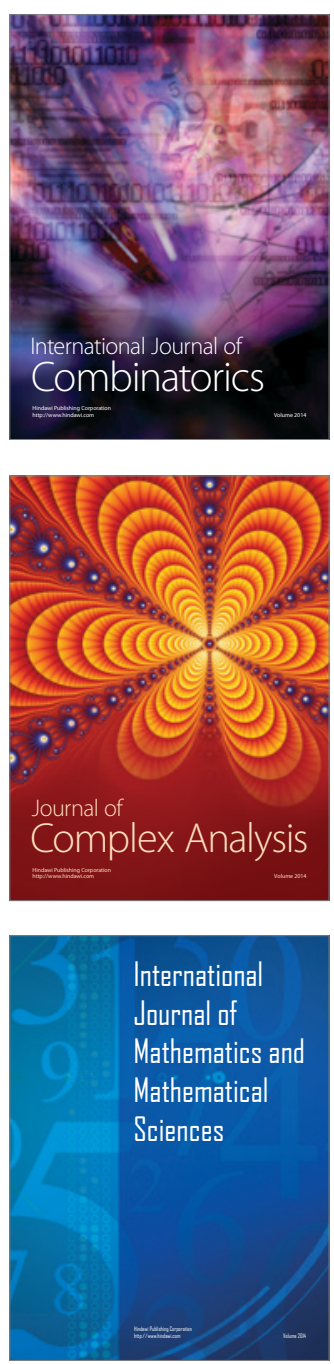
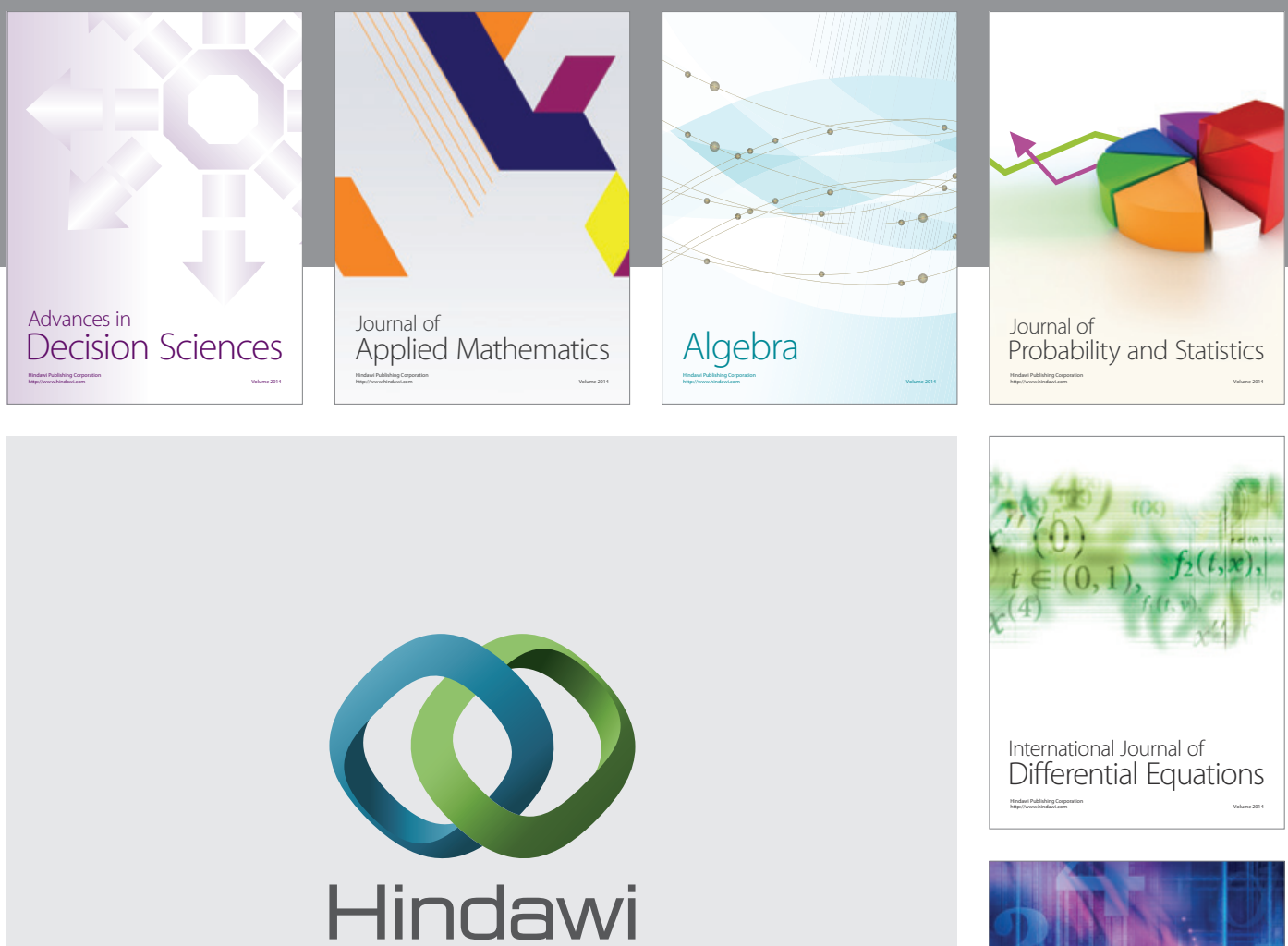

Submit your manuscripts at http://www.hindawi.com
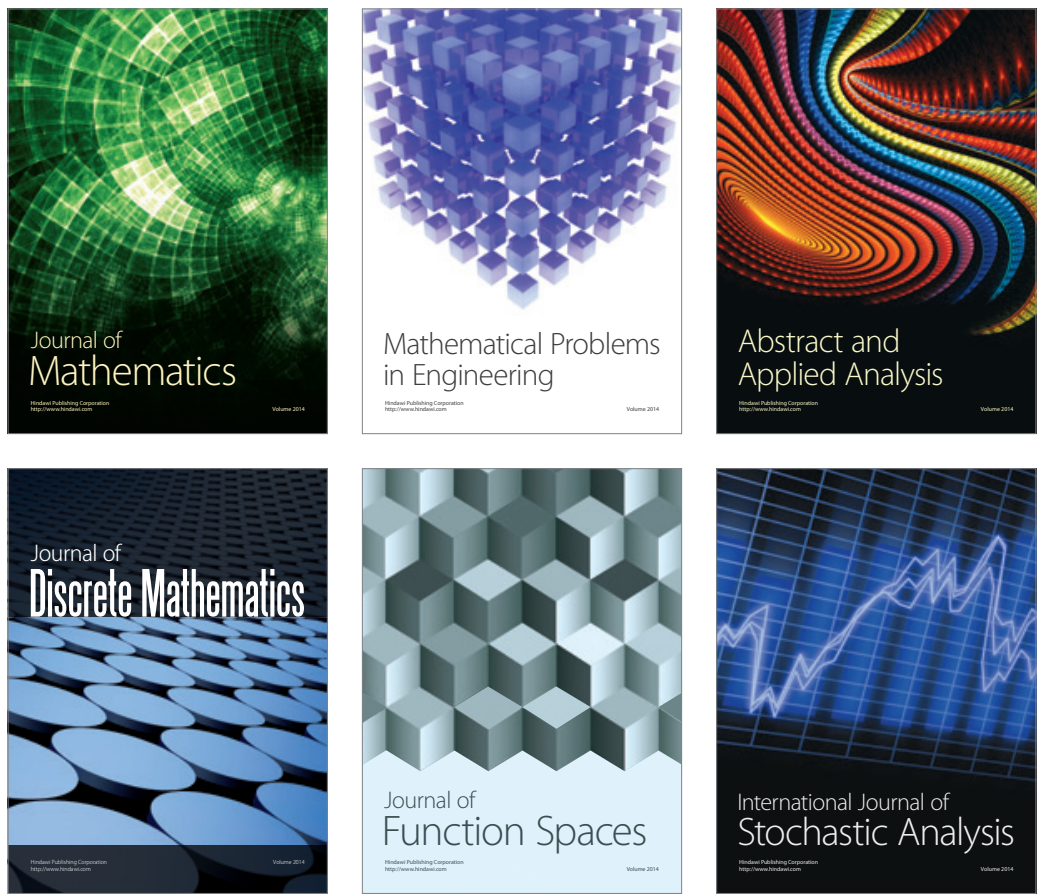

Journal of

Function Spaces

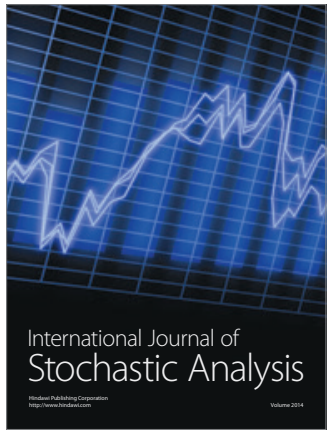

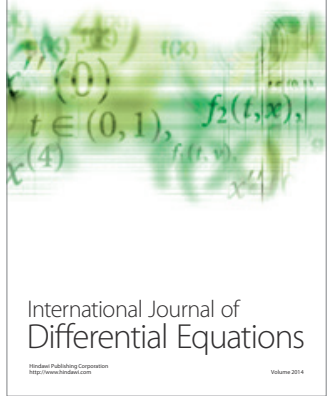
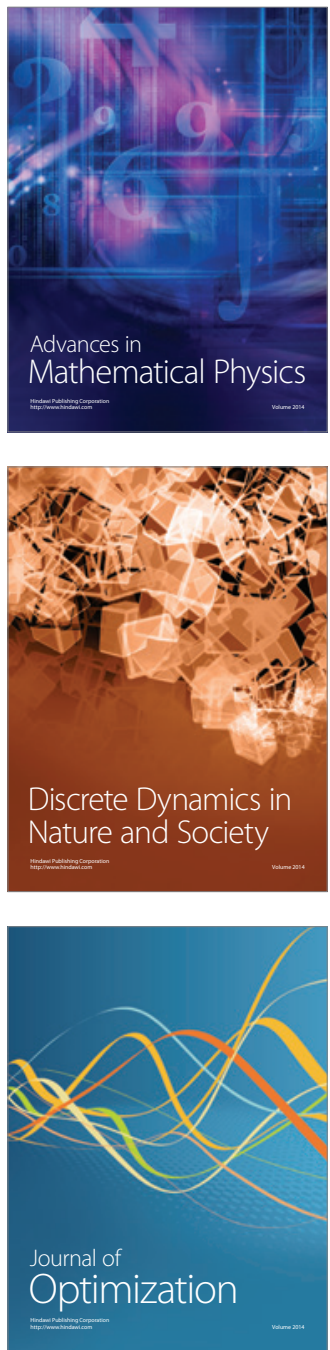